\title{
Bioaccessibility and antioxidant activity of Calendula officinalis supercritical extract as affected by in vitro co-digestion with olive oil
}

Diana Martin ${ }^{1,2, *}$, Joaquín Navarro del Hierro ${ }^{1,2}$, David Villanueva Bermejo ${ }^{1,2}$, Ramon FernandezRuiz $^{3}$, Tiziana Fornari ${ }^{1,2}$, Guillermo Reglero ${ }^{1,2,4}$

${ }^{1}$ Departamento de Producción y Caracterización de Nuevos Alimentos. Instituto de Investigación en Ciencias de la Alimentación (CIAL) (CSIC-UAM), 28049 Madrid, Spain.

${ }^{2}$ Sección Departamental de Ciencias de la Alimentación. Facultad de Ciencias. Universidad Autónoma de Madrid, 28049 Madrid, Spain

${ }^{3}$ Servicio Interdepartamental de Investigación, Laboratorio de Fluorescencia de Rayos X por Reflexión Total (TXRF), Universidad Autónoma de Madrid, 28049 Madrid, Spain.

${ }^{4}$ Imdea-Food Institute. CEI UAM+CSIC, 28049 Madrid, Spain

\footnotetext{
* Corresponding author: Diana Martin. Tel: +34 910017930; Fax: +34 910017905; E-mail: diana.martin@uam.es
} 


\section{ABSTRACT}

1 Supercritical extracts of marigold (ME) were produced and characterized. The bioaccessibility of 2 terpenes, especially that of pentacyclic triterpenes (PT), the particle-size distribution and 3 antioxidant activity after the in vitro co-digestion of ME with olive oil (OO), were determined. ME

4 produced without co-solvent was richer in taraxasterol, lupeol, $\alpha$-amyrin and $\beta$-amyrin than extracts

5 with co-solvent. All terpenes showed high bioaccessibility without OO (>75\%). Significant

6 correlations were found between the molecular properties of compounds ( $\log \mathrm{P}$ and number of

7 rotatable bonds) and their bioaccessibility. Co-digestion with OO enhanced the bioaccessibility

8 (around 100\% for PT), which could be related to a higher abundance of low-size particles of the

9 digestion medium. The antioxidant activity of the digested ME increased around $50 \%$, regardless of

10 OO. PT-rich extracts from marigold display high bioaccessibility and improved antioxidant activity

11 after in vitro digestion, although complete bioaccessibility of PT can be reached by co-digestion

12 with oil, without affecting antioxidant activity.

13

14 Keywords: Calendula officinalis, pentacyclic triterpenes, bioaccessibility, lipid digestion, excipient 15 foods 


\section{INTRODUCTION}

Calendula officinalis L. (common name marigold) belongs to the order of Asterales and is a member of the family Asteraceae. This herbaceous plant, native of the Mediterranean climate areas, is traditionally cultivated in several countries for ornamental, medical and cosmetic purposes. Although it is not extensively known, some edible uses have been described for flowers and leaves. The fresh petals can be chopped and added to salads, curry or custard. The dried petals have a more intense flavor and are used as seasoning in soups, cakes, drinks and baked products. A tea can be prepared from the flowers and petals, and the leaves can be also eaten raw in salads. ${ }^{1-3}$

The traditional medicinal use of the marigold is related to its great variety of phytochemicals of bioactive interest, such as terpenoids, sterols, saponins, carotenoids and phenolic compounds, mainly in flower extracts. Due to this complex composition, the extracts of marigold have been related to activities such as antioxidant, antiinflammatory, immunostimulant, anticancer, hepatoprotective, antimicrobial and wound healing. ${ }^{1-4}$ The triterpenoids as pentacyclic triterpenes (PT) have been described as one of the main responsible for the biological activities of marigold, especially as anti-inflammatory. ${ }^{4}$ The typical PT of the marigold are monohydroxy alcohols ( $\alpha$ amyrin, $\beta$-amyrin, taraxasterol and lupeol), and dihydroxyalcohols (faradiol, arnidiol, brein or calenduladiol).

Taking into account the biological interest of all these compounds, the production of extracts of marigold rich in these bioactive compounds is of current interest. Within the most popular methods for production of plant extracts, the green technology of supercritical fluid extraction (SFE) is quite popular nowadays, with special use in the extraction of compounds with low polarity that are soluble in supercritical $\mathrm{CO}_{2}$, such as the PT. The supercritical $\mathrm{CO}_{2}$ extraction assisted by co-solvents such as ethanol may also enhance the yield of extraction. In the specific case of marigold, diverse studies have reported the use of SFE, ${ }^{5-7}$ but the use of co-solvents has been scarcely explored. ${ }^{7}$ Furthermore, many of them have been focused in the extraction and characterization of the essential oil rich in sesquiterpenes, ${ }^{8-10}$ although the content in PT is not always reported. 
It is important to remark that most of the described bioactive compounds of marigold are compounds of a typical low polarity and high hydrophobicity, which leads to a limitation in their potential use and bioactivity. However, it seems that the available information on the behavior of PT during the gastrointestinal process, bioaccessibility and bioavalability is still scarce and contradictory. It has been suggested that the bioavailability of PT is poor due to a difficult solubilization in the aqueous media of the gastrointestinal tract, necessary for a proper absorption. ${ }^{11}$ However, other studies reported that some PT of fruits and plants were effectively absorbed and deposited in their intact forms in diverse tissues in mice. ${ }^{12}$ In the specific case of the typical PT of marigold, some studies have described that lupeol is bioavailable, ${ }^{13}$ whereas other studies have suggested the opposite. ${ }^{14,15}$ Ching, Lin, Tan $\& \operatorname{Koh}^{16}$ also described a low bioavailability of the amyrin in mice, whereas diverse studies have shown that this compound is orally effective, which would not be in agreement with a poor bioavailability. ${ }^{11,17}$ Concerning other PT from the marigold, such as taraxasterol or faradiol, a lack of information on bioavalability has been found.

One of the factors that might be related to these inconclusive results could be the variability on the composition of the digestion medium. In this respect, for most lipophilic compounds, it has been demonstrated that their bioaccessibility can be improved by the coexistence of other lipids in the intestinal tract. ${ }^{18}$ This is because the lipid digestion leads to the release of fatty acids and monoglycerides that enhance the formation of micellar structures with bile salts and phospholipids. These micelles will include other hydrophobic compounds present in the medium and in turn, the dispersion and absorption of the compounds vehiculized by these micellar structures. In fact, this is one of the fundamentals of current interest in the development of lipid-based delivery systems or the recent term "excipient foods" (a food that increases the bioavailability of bioactive agents that are co-ingested with it), for the improvement in the bioactivity of compounds for food and nutraceuticals. $^{19,20}$ On the other hand, the association of bioactive compounds with lipid components has been suggested as a strategy with other advantages of interest, such as the protection of labile compounds against the conditions of the gastrointestinal tract. ${ }^{21,22}$ 
The aim of the present study was the production of a supercritical extract of Calendula officinalis, in absence and presence of co-solvent, and the characterization on their terpene composition. The subsequent in vitro gastrointestinal digestion of the extract was performed, both in absence and in co-existence with olive oil, in order to evaluate the bioaccessibility and the distribution of particle sizes of the digestion medium. Furthermore, the impact of the gastrointestinal digestion process on the antioxidant activity of the extract was also evaluated.

\section{MATERIALS AND METHODS}

\section{Reagents and Materials}

Dry Calendula officinalis flowers were purchased from a local herbalist supplier (Murcia, Spain). The flowers were ground (particle size smaller than $500 \mu \mathrm{m}$ ) in a knife mill (Grindomix GM200 RETSCH). Extra virgin olive oil was purchased from a local supermarket.

Thymol, $\beta$-caryophillene, valencene, cedrol, $\beta$-sitosterol, tocopherol and alkane mixture (C7-C30) were from Sigma-Aldrich Chemie GmbH (Steinheim, Germany). The standard $\alpha$-amyrin was from Extrasynthese (Genay, France).

Trizma, maleic acid, Amano lipase A from Aspergillus niger, pepsin, pancreatin from porcine pancreas, bile salts, phosphatidyl choline from egg yolk and 2,2-diphenyl-1-picryl-hydrazyl (DPPH) were from Sigma-Aldrich Chemie GmbH (Steinheim, Germany).

\section{Supercritical extraction method}

Extractions were carried out in a pilot-scale supercritical fluid extractor (Thar Technology, Pittsburgh, PA, USA, model SF2000) comprising a 2 L cylindrical extraction cell and two different separators (S1 and S2), each of $0.5 \mathrm{~L}$ capacity, with control of temperature and pressure. The extraction cell was loaded with $400 \mathrm{~g}$ of the milled marigold and the $\mathrm{CO}_{2}$ flow rate was set to 70 $\mathrm{g} / \mathrm{min}$. The extractions were carried out using pure $\mathrm{CO}_{2}$ or $\mathrm{CO}_{2}$ with ethanol as cosolvent $(10 \% \mathrm{p} / \mathrm{p})$. The extraction pressure and temperature were selected at 140 bar and $40{ }^{\circ} \mathrm{C}$ and were kept constant 
95

96

97

98

99

100

101

102

103

104

105

106

107

108

109

110

111

112

113

114

115

116

117

118

119

for all experimental assays. The separator conditions were 40 bar and $40{ }^{\circ} \mathrm{C}$. The total time extraction was $180 \mathrm{~min}$. Samples were stored at $-20{ }^{\circ} \mathrm{C}$ until analysis.

\section{Analysis of terpene compounds of the extracts}

Samples were prepared at $15 \mathrm{mg} / \mathrm{mL}$ in chloroform:methanol $(2: 1, \mathrm{v} / \mathrm{v})$ and were analyzed in an Agilent 7890A system (Agilent Technologies, Santa Clara, CA, USA) comprising a split/splitless injector, an electronic pressure control, a G4513A auto-injector and a 5975C triple-axis mass spectrometer detector. The column used was an Agilent HP-5MS capillary column $(30 \mathrm{~m} \times 0.25$ $\mathrm{mm}$ i.d., $0.25 \mu \mathrm{m}$ phase thickness). Helium was used as carrier gas at $2 \mathrm{~mL} / \mathrm{min}$. The injector temperature was $260{ }^{\circ} \mathrm{C}$ and the mass spectrometer ion source and interface temperatures were 230 and $280{ }^{\circ} \mathrm{C}$, respectively. The sample injections $(1 \mu \mathrm{L})$ were performed in splitless mode. The separation method of Crabas et al. ${ }^{8}$ was used with slight modifications. The oven temperature was initially at $60{ }^{\circ} \mathrm{C}$ and increased to $250{ }^{\circ} \mathrm{C}$ at $4{ }^{\circ} \mathrm{C} / \mathrm{min}$, followed by an increase to $310{ }^{\circ} \mathrm{C}$ at 3 ${ }^{\circ} \mathrm{C} / \mathrm{min}$, and held for $5 \mathrm{~min}$. The mass spectra were obtained by electronic impact at $70 \mathrm{eV}$. The scan rate was $1.6 \mathrm{scan} / \mathrm{s}$ at a mass range of 30-700 amu. Identification of compounds was performed by the NIST MS Data library, the retention indexes of the compounds, the mass spectra according to literature, or according to those of pure commercial compounds whenever possible. Quantitation of compounds was performed by calibration curves obtained from commercial standards whenever possible: thymol was used for monoterpenes, caryophillene and valencene were used for hydrogenated sesquiterpenes, cedrol was used for oxygenated sesquiterpenes, sitosterol was used

for phytosterols, $\alpha$-amyrin was used for PT, and tocopherol was quantitated by its own commercial standard. Other compounds such as alkanes were also quantitated by their own commercial compounds.

\section{In vitro gastrointestinal digestion}


120 The in vitro digestion model was based on Martin, Moran-Valero, Vázquez, Reglero \& Torres ${ }^{23}$

121 with brief modifications and the inclusion of a gastric phase. For gastric digestion, $30 \mathrm{mg}$ of 122 marigold extract (ME) and $10 \mathrm{mg}$ of lecithin were mixed with $14 \mathrm{~mL}$ of a gastric solution $(150 \mathrm{mM}$

$\left.123 \mathrm{NaCl}, 6 \mathrm{mM} \mathrm{CaCl}_{2}, \mathrm{pH} 4.5\right)$. In case of the coexistence of dietary lipids, olive oil (OO) was added at 124 a ratio of ME to oil of 1:2 (w/w). The mixture was placed in an orbital incubator at $200 \mathrm{rpm}$ and 37

$125{ }^{\circ} \mathrm{C}$. After $2 \mathrm{~min}$ of agitation to allow the dispersion of the components, the gastric digestion was 126 initiated by the addition of a fresh extract of gastric enzymes (170 mg of gastric lipase and $15 \mathrm{mg}$ of 127 pepsin in $3 \mathrm{~mL}$ of gastric solution and stirred for $10 \mathrm{~min}$ ). Reaction was continued during $45 \mathrm{~min}$.

128 For intestinal digestion, a solution to simulate biliary secretion was prepared by mixing $0.1 \mathrm{~g}$ of 129 lecithin, $0.25 \mathrm{~g}$ of bile salts, $0.5 \mathrm{~mL}$ of $325 \mathrm{mM} \mathrm{CaCl}_{2}$ solution, $1.5 \mathrm{~mL}$ of $3.25 \mathrm{mM} \mathrm{NaCl}$ solution, 130 and $10 \mathrm{~mL}$ of Trizma-maleate buffer $100 \mathrm{mM} \mathrm{pH} \mathrm{7.5.} \mathrm{This} \mathrm{mixture} \mathrm{was} \mathrm{homogenized} \mathrm{for} 1 \mathrm{~min}$ at $1313500 \mathrm{rpm}$ (Ultra-Turrax IKA T18). Then, the biliary secretion was added to the gastric digestion 132 and shaken in the orbital incubator for $2 \mathrm{~min}$ at $200 \mathrm{rpm}$ and $37^{\circ} \mathrm{C}$. The simulation of intestinal 133 digestion was started by the addition of fresh pancreatin extract $(0.5 \mathrm{~g}$ of pancreatin in $3 \mathrm{~mL}$ of 134 Trizma-maleate buffer, stirred for $10 \mathrm{~min}$ and centrifuged at $1600 \times \mathrm{g}$ for $15 \mathrm{~min})$. Reaction was 135 continued during $60 \mathrm{~min}$. The in vitro digestion of each sample was performed at least in triplicate.

\section{Determination of bioaccessibility}

138 At the end of digestion the medium was submitted to centrifugation at $4000 \mathrm{rpm}$ for $40 \mathrm{~min}$ (5810R

139 Eppendorf Iberica, Madrid, Spain). After centrifugation, an upper aqueous phase and a minor 140 precipitated phase were obtained. The aqueous phase was filtered in order to isolate the aqueous 141 solution containing the micellar structures (micellar phase, MP) from visible and non-solubilized 142 particles of the ME. The components of the ME included in the MP were extracted and analyzed by 143 GC-MS following the same procedure previously described.

144 The bioaccessibility of each compound was determined as the fraction of each compound that was 145 considered available for intestinal absorption, that is, included within the aqueous MP, as: 


\section{Extraction of compounds from the digestion media}

149 At the end of digestion or after isolation of the MP, the components of the ME were extracted with hexane:methyl tert-butyl ether $(50: 50, \mathrm{v} / \mathrm{v})$ at a ratio of $3: 1(\mathrm{v} / \mathrm{v})$ of solvent to sample. The mixture

151 was stirred for $1 \mathrm{~min}$ and centrifuged for $10 \mathrm{~min}$ at $4000 \mathrm{rpm}$ (ScanSpeed mini, Micro Centrifuge). solvent to sample. The two organic phases obtained were mixed and the solvent was removed by rotary evaporator. The obtained extract was solubilized in chloroform:methanol $(2: 1, \mathrm{v} / \mathrm{v})$ at 15 $\mathrm{mg} / \mathrm{mL}$ and analyzed by GC-MS following the same procedure previously described.

\section{Particle size distribution after in vitro digestion}

The particles size distribution of the isolated MP from the in vitro digestion of ME in absence and presence of $\mathrm{OO}$ was measured. Furthermore, the particles size distribution was also determined for two control samples: 1) the MP isolated after in vitro digestion in absence of ME and in absence of $\mathrm{OO}$, and 2) the MP isolated after in vitro digestion in absence of $\mathrm{ME}$ and in presence of OO. By this

162 procedure, it was possible to determine the particles size distribution of the digestion medium itself, in order to distinguish the differences due to the presence of the experimental components. 2000 instrument (Malvern Instruments Ltd., Malvern, Worcestershire, UK), equipped with a dispersion unit of solid particles in liquids (Hydro 2000MU) working at $2000 \mathrm{rpm}$, was used.

167 Trizma-maleate buffer $100 \mathrm{mM} \mathrm{pH} 7.5$ was used as dispersant. Assuming a volume distribution, the

168 largest particle size $\mathrm{D}_{90}$ and the volume mean diameter $\mathrm{D}_{43}$ were the reported parameters. Analyses were performed in quintuplicate. 
172 The antioxidant activity of the digestion media was measured before in vitro digestion and after

173 gastric and intestinal digestion by the DPPH test. The procedure of Martin, Moran-Valero, Casado,

174 Reglero \& Torres $^{24}$ was used with brief modifications. The digestion medium was diluted in 175 methanol:chloroform (5:1 v/v) up to $0.8 \mathrm{mg}$ of $\mathrm{ME} / \mathrm{mL}$. An aliquot $(500 \mu \mathrm{L})$ was added to $1500 \mu \mathrm{L}$ 176 of DPPH in methanol (0.06 mM). Samples were centrifuged at $12000 \mathrm{rpm}$ for $5 \mathrm{~min}$ (miniSpin plus,

177 Eppendorf). Then, reaction was completed after $60 \mathrm{~min}$ at room temperature and darkness, and

178 absorbance was measured at $517 \mathrm{~nm}$. Control experiments of the digestion medium in absence of

179 the tested compounds (ME and OO) were also performed following the same procedure. The 180 remaining DPPH concentration in the reaction medium was estimated by proper calibration curves 181 of DPPH.

182 Antioxidant activity was expressed as percentage of inhibition of DPPH as:

$$
\% \text { inhibition DPPH }=100-\left[\left(\mu \mathrm{g} \mathrm{DPPH} / \mathrm{mL}_{\text {sample }} / \mu \mathrm{g} \mathrm{DPPH} / \mathrm{mL}_{\text {control }}\right) \times 100\right]
$$

\section{Statistical analysis}

Statistical analyses were performed by means of the general linear model procedure of the SPSS 17.0 statistical package (SPSS Inc., Chicago, IL, USA) by one-way analysis of variance. Differences were considered significant at $\mathrm{p} \leq 0.05$. Post-hoc Tukey's tests were performed in order to establish significant differences. Pearson's correlation tests were used to study the bioaccessibility of compounds as related to their molecular properties.

\section{RESULTS AND DISCUSSION}

\section{Characterization of supercritical extracts of Calendula officinalis}

194 The presence or absence of co-solvent in the supercritical extraction of Calendula officinalis led to 195 some differences regarding yield and composition of the extract. The extraction yield of $\mathrm{ME}$ by $\mathrm{CO}_{2}$ 196 in absence of co-solvent was $2.1 \%$, whereas that value was up to $7.5 \%$ in case of ethanol- $\mathrm{CO}_{2}$ 197 extraction. The identified compounds of both extracts are detailed in Table 1 . More than $94 \%$ of the 
198 volatile compounds were identified for both extracts. According to the area percentage, the major

199 abundance corresponded to alkanes (HC), followed by sesquiterpenes (S) and oxygenated 200 triterpenes (OT), in case of extraction without co-solvent. In case of ethanol- $\mathrm{CO}_{2}$ extraction, a slight 201 qualitative difference was found, mainly due to a lower proportion of HC and a higher proportion of 202 OT. However, the major difference due to co-solvent was that the chromatographic areas for all the compounds were lower than the $\mathrm{CO}_{2}$ extraction without co-solvent (Table 1). Taking into account the higher yield of the ethanol- $\mathrm{CO}_{2}$ extraction, this result would suggest that the detected compounds could be diluted with other extracted compounds but non-detected by GC-MS under the used conditions.

In any case, the general composition of both ME was in agreement with previous similar studies about supercritical extracts from this plant. Thus, $\alpha$-cadinol, $\tau$-cadinol, $\gamma$-cadinene and $\delta$-cadinene are typical volatile compounds described for marigold. ${ }^{8,10,25}$ Furthermore, in the current study, the main detected PT were taraxasterol, lupeol, $\alpha$-amyrin and $\beta$-amyrin, as well as diverse nonidentified sterols. The identification of PT from supercritical extracts of marigold has not been usually described in most previous studies, but most of them were focused on the typical terpenes of 213 the essential oil of the extracts.

214 It is important to remark that the relative abundances of the compounds in Table 1 cannot be considered to determine the major compounds of the extracts, due to their differences on chromatographic responses. Therefore, taking into account the importance of characterizing the extract due to its bioactive compounds of interest, especially PT, a proper quantitation as possible was performed for some of the compounds with the available commercial standards. As shown in

219 Table 2, in absence of co-solvent, close to $15 \%$ of the chemical composition of the extract was 220 quantitated, being the OT the most abundant family of compounds. In the specific case of bioactive 221 PT (taraxasterol, lupeol, $\alpha$-amyrin and $\beta$-amyrin), these compounds were close to $3 \%$ of the extract. 222 Furthermore, up to $5 \%$ of the extract was also quantitated as non-identified sterols. 
223 Regarding the ethanol- $\mathrm{CO}_{2}$ extract, due to the dilution effect with other extracted but non-identified 224 compounds, the total amount of quantitated compounds by GC-MS was lower than 5\%, but 225 similarly to the $\mathrm{CO}_{2}$ extract, the major compounds were the OT (4\%) and the bioactive PT were 226 around $1.5 \%$ (Table 2$)$.

227 As summary, both supercritical ME might be considered of interest due to their content on bioactive 228 PT, although the extraction of marigold in absence of co-solvent resulted in the preferred procedure. 229 Therefore, taking into account the interest of the bioactive PT, the following studies were performed 230 with the ME produced in absence of co-solvent.

\section{Bioaccessibility of supercritical extract of Calendula officinalis}

233 After in vitro digestion of the ME, the bioaccessibility of the major quantitated compounds of the 234 extract was determined. Due to the complex composition of the extract, as well as the increased complexity of the chromatographic analyses due to the co-elution of other compounds from the digestion medium itself, a selection of compounds for the study of bioaccessibility was performed. Such selection was based on the preferential characterization of the bioactive compounds of interest (PT), as well as the consideration of other representative compounds of each chemical family of the extract $(\mathrm{SH}, \mathrm{OS}$ and $\mathrm{HC})$ that were present in the extract at concentration $\geq 0.1 \%$ at least. The selected compounds and their values of bioaccessibility are shown in Table 3.

According to Table 3, the bioaccessible fraction of the compounds was quite variable, since percentages higher than $80 \%$ were found for some compounds, whereas other compounds showed values lower than $50 \%$. In general, it seemed that the bioaccessibility of the family of compounds

244 decreased in the following order: $\mathrm{SH}(84 \%)>\mathrm{OS}(81 \%)>\mathrm{OT}(77 \%)>\mathrm{HC}(42 \%)$. Thus, it could be 245 considered that most terpenes, included the PT, showed a high bioaccessibility, since more than $75 \%$ of the amount of terpenes were found within the bioaccessible MP, including the bioactive PT

247 (Table 3). The available information about the bioaccessibility of terpenes in general is scarce, and 248 bioaccessibility values of PT in particular has not been found in the scientific literature, whereas 
249

250

251

252

253

254

255

256

257

258

259

260

261

262

263

264

265

266

267

268

269

270

271

272

273

274

contradictory results have been reported about the bioavalability of compounds such as lupeol or amyrins. ${ }^{11,13-17}$ According to the obtained results, the present study showed that the studied PT of a supercritical extract of ME might have high bioaccessibility.

Diverse reasons might be considered to explain the observed results. In general, the bioaccessibility of compounds in the aqueous medium of the intestinal lumen is determined by their solubility. ${ }^{26,27}$ This solubility is not a problem for those compounds with a good hydrosolubility or hydrophilic/lipophilic balance that ensure its dispersion in the medium, either directly, or indirectly, by inclusion in vesicles, emulsion droplets, lamellar or micellar structures of bile salts and phospholipids naturally present in the intestinal tract. In fact, absorption of lipophilic products takes place supported by these structures of the MP, which enhances the transport of such products to enterocytes through the unstirred water layer close to the microvillous membrane, where they are absorbed. ${ }^{26,27}$ According to this theory, most of terpenes might show a proper dispersion in the aqueous media after in vitro digestion. On the contrary, alkanes might not be so effectively dispersed within the medium and hence their lower bioaccessibility.

In order to understand whether the obtained results were related to the lipophilicity of the compounds, the $\log \mathrm{P}$ value of each individual substance listed in Figure 1 was considered (Food Database FooDB, www.foodb.ca). As it is illustrated in Figure 1, a negative correlation was found between the $\log \mathrm{P}$ value of the compounds and their bioaccessibility $(r=-0.771, \mathrm{P}<0.001)$. Therefore, the higher lipophilicity of the compounds, the worse the bioaccessibility is. Thus, $\log \mathrm{P}$ values lower than 8 might be preferred for a high bioaccessibility (closer to 80\%). All PT identified in ME showed $\log \mathrm{P}$ values around 6.

Another molecular property that has been popularly related to the bioavailability of drugs is the molecular flexibility, due to the number of rotatable bonds (NRB) described by Veber, Johnson, Cheng, Smith, Ward \& Kopple ${ }^{28}$. A low NRB value has been suggested as one strong criterion for drug candidates with proper bioavailability, although the exact reason for such relation has not been established. According to Figure 2, a strong negative correlation was found between the NRB value 
275 of the studied compounds (Molinspiration Cheminformatics, Bratislava, Slovak Republic) and their 276 bioaccessibility $(r=-0.860, \mathrm{P}<0.001)$. Therefore, the higher the molecular flexibility, the worse 277 the bioaccessibility is. Thus, NRB values of 0 or 1 might be desirable for a high bioaccessibility of 278 the studied compounds. This was considered an interesting result, since previous information on the 279 relation between the molecular flexibility and bioaccessibility of compounds has not been 280 described, but only the relation of NRB with bioavailability.

281 Therefore, according to the obtained results, the compounds of bioactive interest of the ME showed 282 high bioaccessibilities that might be related to their favorable molecular properties. At any case, 283 further studies at this respect would be necessary in order to understand whether such proper 284 bioaccessibility might lead to a positive bioavailability and bioactivity.

Bioaccessibility of Calendula officinalis supercritical extract co-digested with olive oil

Despite that the studied compounds showed a proper bioaccessibility, it was considered interesting the study of the role of the coexistence of lipids (olive oil, OO) during the digestion process in order to evaluate whether it would be possible to reach a complete bioaccessibility of the bioactive compounds of interest. Preliminary studies were performed in order to find the best ratio ME to oil that allowed the best bioaccessibility for most compounds (data not shown). This ratio was established as 1:2(w/w) and the corresponding results are shown in Figure 3. In general, a higher bioaccessibility due to the $\mathrm{OO}$ factor was found $(\mathrm{P}<0.001)$. The different chemical families increased around $20 \%$ their bioaccessibility, and most compounds of interest reached values of bioaccessibility closer to $100 \%$.

During lipid digestion, the major hydrolysis products as fatty acids and monoglycerides are 297 released. These compounds lead to the formation of micellar structures with bile salts and 298 phospholipids, which is necessary for the proper absorption of fats by enterocytes. ${ }^{27}$ This increase 299 in the micellar surface compared to the absence of oil increases the available structures for inclusion 300 of other hydrophobic compounds present in the aqueous media and hence, their bioaccessibility is 
301 enhanced. ${ }^{18-20}$ This mechanism would be related to the results obtained in the present study.

302 Therefore, the current study showed that despite the bioaccessibility of bioactive compounds such

303 as PT from a supercritical extract of marigold was high; the co-digestion with particularly low

304 levels of a typical dietary fat would be enough to reach a complete bioaccessibility of such

305 compounds. In this respect, according to the term of "excipient food" recently described by

306 McClements et al., ${ }^{19}$ as a food that increases the bioavailability of bioactive agents that are co-

307 ingested with it, olive oil might be a potential candidate as "excipient food" to enhance the

308 bioaccessibility of compounds of ME in general, and of bioactive PT in particular. As far as we

309 know, previous studies about the effect of coexistence of oils on the gastrointestinal digestion

310 behavior and bioaccessibility of PT have not been described in the scientific literature.

\section{Particle size distribution after in vitro digestion}

313 The particle size distribution of the isolated MP was characterized in order to deepen the 314 understanding of the hypothesis that a better bioaccessibility of the compounds was due to an

315 enhanced dispersion by digested lipids. Previously, we considered necessary to understand the 316 typical particles size distribution of the own MP in absence of any of the experimental compounds, 317 that is, in absence of ME and OO. As shown in Figure 4.a, there were two major peaks, one in the 318 range of $0.2 \mu \mathrm{m}$, and a second one in the range of $1 \mu \mathrm{m}$. Furthermore, another abundant volume of 319 particles was found within a wide range of sizes between 4 and $240 \mu \mathrm{m}$. This distribution led to a 320 MP characterized by particles most of them lower than $25 \mu \mathrm{m}\left(\mathrm{D}_{90}\right)$ and a volume mean diameter 321 around $11 \mu \mathrm{m}\left(\mathrm{D}_{43}\right)$. It is complicated to determine the precise components of the medium 322 responsible of such distribution, but it might probably be related to the particles formed by 323 phospholipids, bile salts, either individually or in combination as micelles or vesicles. ${ }^{29}$

324 When OO was digested, the particle size distribution of the MP changed (Figure 4.b). The typical 325 modes at 0.2 and $1 \mu \mathrm{m}$ were also present, but the area of the mode at $0.2 \mu \mathrm{m}$ was higher, whereas 326 the modes at 1 and 4-240 $\mu \mathrm{m}$ were much lower. Thus, the MP was characterized by particles most 
327 of them lower than $2 \mu \mathrm{m}\left(\mathrm{D}_{90}\right)$ and a volume mean diameter around $7 \mu \mathrm{m}\left(\mathrm{D}_{43}\right)$. The obtained 328 results would suggest that the hydrolysis products of $\mathrm{OO}$, mainly fatty acids and monoglycerides, 329 contributed to an increase in the number of particles of lower size within the MP. These observed 330 results were quite useful since they could confirm that the in vitro digestion model led to a 331 physiological and favorable situation to enhance the dispersion of other lipophilic compounds in the 332 aqueous media.

333 When the ME was digested in absence of OO, the typical modes of the medium at 0.2 and $1 \mu \mathrm{m}$ 334 were present again; however, a relevant decrease was produced at the expense of an increase in the 335 abundance of bigger particles (Figure 4.c). In fact, three new modes of particles appeared in the 336 range of $6 \mu \mathrm{m}, 65 \mu \mathrm{m}$ and $500 \mu \mathrm{m}$. Thus, in presence of ME, the MP was characterized by bigger 337 particles, because the $10 \%$ of particles were even higher than $190 \mu \mathrm{m}\left(\mathrm{D}_{90}\right)$ and the volume mean 338 diameter was around $47 \mu \mathrm{m}\left(\mathrm{D}_{43}\right)$. This distribution was quite different compared to the control 339 samples (Figure 4.a and 4.b). Therefore, it could be thought that the obtained results might be 340 mainly related to particles of the ME dispersed in the aqueous media, either in isolation or by 341 interacting with components of the medium.

342 When the ME was digested in co-existence with OO, the basic modes of the medium at 0.2 and 1 $343 \mu \mathrm{m}$ increased again and the big particle sizes previously observed for the ME sample decreased at 344 the expense of the formation of a wide mode in the range of 15-200 $\mu \mathrm{m}$ (Figure 4.d). Thus, in 345 presence of $\mathrm{OO}$, the MP from the digestion of ME was characterized by lower particles than in 346 absence of OO, being most of them lower than $28 \mu \mathrm{m}\left(\mathrm{D}_{90}\right)$ and a volume mean diameter around 9 $347 \mu \mathrm{m}\left(\mathrm{D}_{43}\right)$. Those values were closer to those obtained for the control sample of MP in absence of 348 ME and OO (Figure 4.a). This might confirm our proposed theory that hydrolysis products of lipids 349 could enhance the dispersion of hydrophobic compounds of ME, by increasing the number of lower 350 size particles.

351 Therefore, the study of the distribution of particle sizes after in vitro digestion of ME showed that 352 the co-digestion of the extract with low levels of a typical dietary fat might enhance the dispersion 
353 of the marigold components within the aqueous phase of the intestinal medium and, in turn, would

354 enhance its bioaccessibility (Table 3). As far to our knowledge, previous studies on the particle size 355 distribution of the aqueous medium after digestion of ME, either with or without oils, have not been 356 described in the scientific literature. In any case, further studies would be necessary in order to 357 confirm the observed evidences, taking into account that the diluting and stirring conditions 358 commonly used for particle size measurement might lead to the formation of artefacts.

\section{Antioxidant activity of Calendula officinalis supercritical extract during in vitro digestion}

361 The modification of the antioxidant activity of diverse compounds, either negatively or positively, 362 after the process and conditions of gastrointestinal digestion has been previously described. In case 363 of a detrimental effect after digestion, the association of bioactive compounds with lipid components has been reported as a strategy to protect those labile compounds from the conditions

365 of the gastrointestinal tract. ${ }^{21,22}$ Therefore, taking into account that some components of marigold 366 have been described as antioxidants, it was considered interesting the study of the impact of the 367 digestion process on such activity, either in absence or in presence of olive oil.

368 As shown in Figure 5, the ability of the ME to inhibit the DPPH radical significantly increased with 369 the course of the gastrointestinal digestion $(\mathrm{P}=0.006)$. Such increase was especially significant after 370 intestinal digestion for both treatments. Furthermore, the final inhibitory activity that was observed 371 after intestinal digestion was quite similar between treatments, regardless of the presence or absence 372 of OO. Thus, the inhibitory activity of ME increased after gastrointestinal digestion around $50 \%$ 373 and $40 \%$ for the treatments in absence and presence of OO, respectively. The improvement of the 374 antioxidant activity of compounds during the gastrointestinal process has been previously described, 375 especially for polyphenols, due to their release during the hydrolysis processes from other complex 376 molecules. ${ }^{30}$ As far as we know, previous information about the antioxidant activity of compounds 377 from ME as affected by gastrointestinal digestion has not been described. 
378 Therefore, the observed results showed that the antioxidant effect of ME was not negatively 379 affected, but rather enhanced by the in vitro gastrointestinal process. Additionally, it seemed that 380 the better dispersion of the extract that was found in presence of OO was not related to these results, 381 at least in the case of intestinal digestion, since during gastric digestion the antioxidant activity was 382 significantly higher in presence of OO. Other in vitro and in vivo studies would be necessary in 383 order to evaluate whether these preliminary results would be related to enhanced antioxidant 384 activities of a digested ME.

385 As a summary, the present study showed that supercritical extraction in absence of co-solvent is the 386 preferred procedure for producing a marigold extract rich in bioactive compounds such as 387 pentacyclic triterpenes. Although that such bioactive compounds show a good bioaccessibility, it 388 can be even improved by the co-digestion with particularly low levels of a typical dietary fat such as olive oil, thanks to a better dispersion of the extract in the aqueous media during gastrointestinal digestion. Additionally, the gastrointestinal process enhances the antioxidant activity of the extract, 391 regardless of the co-digestion with olive oil. The obtained results are of interest either to obtaining a 392 deeper knowledge on the potential of the marigold plant as a possible bioactive ingredient of foods, 393 as well as to contributing to the general knowledge on the gastrointestinal digestion of bioactive 394 compounds such as pentacyclic triterpenes, together with other typical compounds of supercritical extracts of plants in general, such as sesquiterpenes or alkanes.

PT Pentacyclic triterpenes

OO Olive oil

SFE Supercritical fluid extraction

MP Micellar phase

HC Alkanes 
Sesquiterpenes

SH Sesquiterpenes hydrocarbons

OT Oxygenated triterpenes

OS Oxygenated sesquiterpenes

NRB Number of rotatable bonds

398

\section{ACKNOWLEDGMENTS}

400 Joaquín Navarro del Hierro thanks the Universidad Autónoma de Madrid for funding his 401 collaboration scholarship during his Official Master's Degree studies.

402 
403

404

405

406

407

408

409

410

\section{REFERENCES}

1. Lim, T.K. Calendula officinalis. In Edible Medicinal and Non-Medicinal Plants; Lim, T.K., Ed; Springer Science+Business Media: The Netherlands, 2014; Vol. 7; pp. 213-244.

2. Mubashar Sabir, S.; Khan, M. F.; Rocha, J. B. T.; Boligon, A. A.; Athayde, M. L. Phenolic Profile, Antioxidant Activities and Genotoxic Evaluations of Calendula officinalis. J. Food. Biochem. 2015, 39, 316-324.

3. Benvenuti, S.; Bortolotti, E.; Maggini, R. Antioxidant power, anthocyanin content and organoleptic performance of edible flowers. Sci. Hortic-Amsterdam. 2016, 199, 170-177.

4. Dall'Acqua, S.; Catanzaro, D.; Cocetta, V.; Igl, N.; Ragazzi, E.; Giron, M. C.; Cecconello, L.; Montopoli, M. Protective effects of $\psi$ taraxasterol 3-O-myristate and arnidiol 3-O-myristate isolated from Calendula officinalis on epithelial intestinal barrier. Fitoterapia. 2016, 109, 230-235.

5. Hamburger, M.; Adler, S.; Baumann, D.; Förg, A.; Weinreich, B. Preparative purification of the major anti-inflammatory triterpenoid esters from Marigold (Calendula officinalis). Fitoterapia. 2003, 74, 328-338.

6. Baumann, D.; Adler, S.; Grüner, S.; Otto, F.; Weinreich, B.; Hamburger, M. (2004). Supercritical carbon dioxide extraction of marigold at high pressures: comparison of analytical and pilot-scale extraction. Phytochem. Analysis. 2004, 15, 226-230.

7. Palumpitag, W.; Prasitchoke, P.; Goto, M.; Shotipruk, A. Supercritical carbon dioxide extraction of marigold lutein fatty acid esters: Effects of cosolvents and saponification conditions. Separ. Sci. Technol. 2011, 46, 605-610.

8. Crabas, N.; Marongiu, B.; Piras, A.; Pivetta, T.; Porcedda, S. Extraction, separation and isolation of volatiles and dyes from Calendula officinalis L. and Aloysia triphylla (L'Her.) Britton by supercritical CO2. J. Essent. Oil Res. 2003, 15, 272-277.

9. Danielski, L.; Campos, L. M.; Bresciani, L. F.; Hense, H.; Yunes, R. A.; Ferreira, S. R. Marigold (Calendula officinalis L.) oleoresin: solubility in SC-CO 2 and composition profile. Chem Eng Process. 2007, 46, 99-106. 
10. Petrović, L.; Lepojević, Ž.; Sovilj, V.; Adamović, D.; Tešević, V. Composition of essential oil obtained from tubular, head and ligulate flowers of Calendula officinalis L. by steam distillation of plant material and CO2 extracts. J. Essent. Oil Res. 2010, 22, 143-146.

11. Santos, F. A.; Frota, J. T.; Arruda, B. R.; de Melo, T. S.; de Castro Brito, G. A.; Chaves, M. H.; $\underline{\text { Rao, V. S. Antihyperglycemic and hypolipidemic effects of } \alpha, \beta \text {-amyrin, a triterpenoid mixture }}$ from Protium heptaphyllum in mice. Lipids Health Dis. 2012, 11, 98-106.

12. Yin, M. C.; Lin, M. C.; Mong, M. C.; Lin, C. Y. Bioavailability, distribution, and antioxidative effects of selected triterpenes in mice. J. Agric. Food. Chem. 2012, 60, 7697-7701.

13. Siddique, H. R.; Mishra, S. K.; Karnes, R. J.; Saleem, M. Lupeol, a novel androgen receptor inhibitor: implications in prostate cancer therapy. Clin. Cancer Res. 2011, 17, 5379-5391.

14. Cháirez-Ramírez, M. H.; Sánchez-Burgos, J. A.; Gomes, C.; Moreno-Jiménez, M. R.; GonzálezLaredo, R. F.; Bernad-Bernad, M. J.; Medina-Torres, L.; Ramírez-Mares, M.V.; Gallegos-Infante, J.A.; Rocha-Guzmán, N. E. Morphological and release characterization of nanoparticles formulated with poly (dl-lactide-co-glycolide)(PLGA) and lupeol: In vitro permeability and modulator effect on NF-кB in Caco-2 cell system stimulated with TNF- $\alpha$. Food Chem. Toxicol. 2015, 85, 2-9.

15. Wang, W. H.; Chuang, H. Y.; Chen, C. H.; Chen, W. K.; Hwang, J. J. Lupeol acetate ameliorates collagen-induced arthritis and osteoclastogenesis of mice through improvement of microenvironment. Biomed. Pharmacother. 2016, 79, 231-240.

16. Ching, J.; Lin, H. S.; Tan, C. H.; Koh, H. L. Quantification of $\alpha$-and $\beta$-amyrin in rat plasma by gas chromatography-mass spectrometry: application to preclinical pharmacokinetic study. J. Mass Spectrom. 2011, 46, 457-464.

17. Melo, C. M.; Morais, T. C.; Tomé, A. R.; Brito, G. A. C.; Chaves, M. H.; Rao, V. S.; Santos, F. A. Anti-inflammatory effect of $\alpha, \beta$-amyrin, a triterpene from Protium heptaphyllum, on ceruleininduced acute pancreatitis in mice. Inflamm. Res. 2011, 60, 673-681.

18. Gupta, S.; Kesarla, R.; Omri, A. Formulation strategies to improve the bioavailability of poorly absorbed drugs with special emphasis on self-emulsifying systems. ISRN Pharm. 2013, 848043. 
455

456

457

458

459

460

461

462

463

464

465

466

467

468

469

470

471

472

473

474

475

476

477

478

19. McClements, D. J.; Zou, L.; Zhang, R.; Salvia-Trujillo, L.; Kumosani, T.; Xiao, H. Enhancing nutraceutical performance using excipient foods: designing food structures and compositions to increase bioavailability. Compr. Rev. Food Sci. Food Saf. 2015, 14, 824-847.

20. Aboalnaja, K. O.; Yaghmoor, S.; Kumosani, T. A.; McClements, D. J. Utilization of nanoemulsions to enhance bioactivity of pharmaceuticals, supplements, and nutraceuticals: Nanoemulsion delivery systems and nanoemulsion excipient systems. Expert Opin. Drug Deliv. 2016, 21, 1-10.

21. Mohsin, K.; Shahba, A. A.; Alanazi, F. K. Lipid based self emulsifying formulations for poorly water soluble drugs-an excellent opportunity. Indian J. Pharm. Educ. 2012, 46, 88-196.

22. Yao, M.; McClements, D. J.; Xiao, H. Improving oral bioavailability of nutraceuticals by engineered nanoparticle-based delivery systems. Curr. Opin. Food Sci. 2015, 2, 14-19.

23. Martin, D.; Moran-Valero, M. I.; Vázquez, L.; Reglero, G.; Torres, C. F. Comparative in vitro intestinal digestion of 1, 3-diglyceride and 1-monoglyceride rich oils and their mixtures. Food Res. Int. 2014, 64, 603-609.

24. Martin, D.; Moran-Valero, M. I.; Casado, V.; Reglero, G.; Torres, C. F. Phosphatidyl Derivative of Hydroxytyrosol. In Vitro Intestinal Digestion, Bioaccessibility, and Its Effect on Antioxidant Activity. J. Agric. Food. Chem. 2014, 62, 9751-9759.

25. Muley, B. P.; Khadabadi, S. S.; Banarase, N. B. Phytochemical constituents and pharmacological activities of Calendula officinalis Linn (Asteraceae): a review. Trop. J. Pharm. Res. 2009, 8, 455-465.

26. Porter, C.; Charman, W. In vitro assessment of oral lipid based formulations. Adv. Drug Deliv. Rev. 2001, 50, s127-s147.

27. Ramirez, M.; Amate, L.; Gil, A. Absorption and distribution of dietary fatty acids from different sources. Early Hum. Dev. 2001, 65, s95-s101. 
479 28. Veber, D. F.; Johnson, S. R.; Cheng, H. Y.; Smith, B. R.; Ward, K. W.; Kopple, K. D.

480 Molecular properties that influence the oral bioavailability of drug candidates. J. Med. Chem. 2002, $481 \quad 45,2615-2623$.

482 29. Zhang, Z.; Zhang, R.; Zou, L.; Chen, L.; Ahmed, Y.; Al Bishri, W.; Khadija, B.; McClements, 483 D. J. Encapsulation of curcumin in polysaccharide-based hydrogel beads: Impact of bead type on 484 lipid digestion and curcumin bioaccessibility. Food Hydrocoll. 2016, 58, 160-170.

485 30. Akillioglu, H. G.; Karakaya, S. Changes in total phenols, total flavonoids, and antioxidant 486 activities of common beans and pinto beans after soaking, cooking, and in vitro digestion process. 487 Food Sci. Biotechnol., 2010, 19, 633-639.

488

$489 \quad$ Funding

490 This work was supported by the Ministerio de Economía y Competitividad, Spain (AGL2013491 48943-C2-1-R) and the Community of Madrid, Spain (ALIBIRD-CM S2013/ABI-2728).

492

$493 \quad$ Notes

494 The authors declare no competing financial interest. 495 
496

497

498

499

500

501

502

503

504

505

506

507

508

509

510

511

512

513

514

515

516

517

518

519

\section{6}

\section{Figure Captions}

Figure 1. Correlation between lipophilicity $(\log \mathrm{P})$ of compounds from supercritical marigold extract and their bioaccessibility $(\%)$

Figure 2. Correlation between molecular flexibility (NRB) of compounds from supercritical marigold extract and their bioaccessibility (\%)

Figure 3. Bioaccessibility (\%) of compounds from supercritical marigold extract as affected by olive oil during in vitro digestion. Bars within the same compound are significantly different if $\mathrm{p} \leq$ $0.05(*), \mathrm{p} \leq 0.01(* *)$ or $\mathrm{p} \leq 0.001(* * *)$

Figure 4. Volume particle size distribution of the digestion media after in vitro digestion. Isolated aqueous micellar phase after in vitro digestion of a) without marigold and without olive oil, b) without marigold and with olive oil, c) with marigold and without olive oil, and d) with marigold and with olive oil.

Figure 5. Evolution of the antioxidant activity of supercritical marigold extract throughout in vitro digestion. Different letters within the same treatment are significantly different. Bars within the same color are significantly higher if $\mathrm{p} \leq 0.05(*)$ or $\mathrm{p} \leq 0.001(* * *)$. 
Table 1. GC-MS Characterization of Supercritical Extracts of Calendula Officinalis

\begin{tabular}{|c|c|c|c|c|c|}
\hline \multirow[t]{2}{*}{ RI } & \multirow[t]{2}{*}{ Compound } & \multicolumn{2}{|c|}{$\mathrm{CO}_{2}$} & \multicolumn{2}{|c|}{ Ethanol- $\mathrm{CO}_{2}$} \\
\hline & & Area & $\%$ & Area & $\%$ \\
\hline 1295 & Thymol & 1535567 & 0.74 & 414731 & 0.91 \\
\hline 1304 & Carvacrol & 613051 & 0.30 & 185772 & 0.41 \\
\hline 1351 & $\alpha$-Cubebene & 416655 & 0.20 & 105666 & 0.23 \\
\hline 1378 & $\alpha$-Copaene & 968862 & 0.47 & 272124 & 0.60 \\
\hline 1391 & $\beta$-Cubebene & 625195 & 0.30 & 113123 & 0.25 \\
\hline 1430 & $\beta$-Gurjunene & 481604 & 0.23 & 69838 & 0.15 \\
\hline 1442 & $\beta$-Humulene & 95765 & 0.05 & 96099 & 0.21 \\
\hline 1464 & Alloaromadendrene & 898623 & 0.43 & 163108 & 0.36 \\
\hline 1478 & y-Muurolene & 901727 & 0.43 & 248135 & 0.55 \\
\hline 1487 & $\beta$-Ionone & 617759 & 0.30 & 119421 & 0.26 \\
\hline 1490 & $\beta$-Selinene & 363937 & 0.18 & 33297 & 0.07 \\
\hline 1497 & $(+)$-Ledene & 2385902 & 1.15 & 495858 & 1.09 \\
\hline 1502 & $\alpha$-Muurolene & 1756737 & 0.85 & 464359 & 1.02 \\
\hline 1516 & y-Cadinene & 5778816 & 2.78 & 2006429 & 4.42 \\
\hline 1525 & $\delta$-Cadinene & 6024229 & 2.90 & 1726232 & 3.80 \\
\hline 1528 & Dihydroactinidiolide & 2694113 & 1.30 & 795656 & 1.75 \\
\hline 1535 & Cadina-1(2),4-diene & 1330919 & 0.64 & 115862 & 0.25 \\
\hline 1539 & $\alpha$-Cadinene & 1086998 & 0.52 & 249607 & 0.55 \\
\hline 1544 & $\alpha$-Calacorene & 408864 & 0.20 & 61864 & 0.14 \\
\hline 1593 & Viridifloror & 3118592 & 1.50 & 389411 & 0.86 \\
\hline 1610 & 1-10-di-epi-cubenol & 805223 & 0.39 & 212891 & 0.47 \\
\hline 1616 & $\delta$-Cadinol & 749509 & 0.36 & 151504 & 0.33 \\
\hline 1629 & Cubenol & 824483 & 0.40 & 190310 & 0.42 \\
\hline 1645 & $\tau$-Cadinol & 10030609 & 4.83 & 2369705 & 5.22 \\
\hline 1648 & n.i. oxygenated sesquiterpene & 1062037 & 0.51 & 264170 & 0.58 \\
\hline 1652 & $\beta$-Eudesmol & 2101899 & 1.01 & 430441 & 0.95 \\
\hline 1658 & $\alpha$-Cadinol & 12114169 & 5.83 & 2456127 & 5.41 \\
\hline 1670 & n.i. ${ }^{a}$ & 1095520 & 0.53 & 218458 & 0.48 \\
\hline 1711 & 3-Hydroxy-5,6-epoxy- $\beta$-ionone & 1793930 & 0.86 & n.d. ${ }^{b}$ & n.d. \\
\hline 1740 & $\begin{array}{l}\text { 1-cyclohexanone, 2-methyl-2-(3- } \\
\text { methyl-2-oxobutyl) }\end{array}$ & 10426982 & 5.02 & 2808684 & 6.18 \\
\hline 1779 & $9,10-$ & 16481274 & 7.93 & 5541526 & 12.20 \\
\hline
\end{tabular}


dimethyltricyclo[4.2.1.1.(2,5)]decane-

9,10-diol

\begin{tabular}{|c|c|c|c|c|c|}
\hline 1840 & n.i. & 857747 & 0.41 & n.d. & n.d. \\
\hline 1846 & Hexahydrofarnesyl acetone & 4377593 & 2.11 & n.d. & n.d. \\
\hline 1901 & Nonadecane & 4110435 & 1.98 & 862927 & 1.90 \\
\hline 1970 & Verticiol & 2312239 & 1.11 & 547645 & 1.21 \\
\hline 1996 & Palmitic acid, ethyl ester & 315697 & 0.15 & 111172 & 0.24 \\
\hline 2001 & Eicosane & 980904 & 0.47 & 163966 & 0.36 \\
\hline 2101 & Heneicosane & 6802339 & 3.27 & 1116421 & 2.46 \\
\hline 2201 & Docosane & 630054 & 0.30 & 104863 & 0.23 \\
\hline 2302 & Tricosane & 8930569 & 4.30 & 1385382 & 3.05 \\
\hline 2402 & Tetracosane & 1111476 & 0.54 & 148787 & 0.33 \\
\hline 2503 & Pentacosane & 13651831 & 6.57 & 1894387 & 4.17 \\
\hline 2601 & Hexacosane & 980678 & 0.47 & 89782 & 0.20 \\
\hline 2708 & Heptacosane & 18232039 & 8.78 & 1943518 & 4.28 \\
\hline 2797 & Octacosane & 2050351 & 0.99 & 242332 & 0.53 \\
\hline 2892 & Nonacosane & 19427953 & 9.35 & 2277964 & 5.01 \\
\hline 2978 & Triacontane & 2191196 & 1.05 & n.d. & n.d. \\
\hline- & Hentriacontane & 11165954 & 5.38 & 1739227 & 3.83 \\
\hline- & $\alpha$-Tocopherol & 827910 & 0.40 & 442105 & 0.97 \\
\hline- & Dotriacontane & 180722 & 0.09 & n.d. & n.d. \\
\hline- & n.i. sterol & 175177 & 0.08 & n.d. & n.d. \\
\hline- & n.i. sterol & 728069 & 0.35 & 540417 & 1.19 \\
\hline- & n.i. sterol & 2017797 & 0.97 & 710705 & 1.56 \\
\hline- & $\beta$-Amyrenone & 578061 & 0.28 & 262928 & 0.58 \\
\hline- & $\beta$-Amyrin & 2940308 & 1.42 & 1433928 & 3.16 \\
\hline- & n.i. sterol & 595111 & 0.29 & 287959 & 0.63 \\
\hline- & $\alpha$-Amyrin + Lupeol & 4327976 & 2.08 & 2095993 & 4.61 \\
\hline- & n.i. oxygenated triterpene & 643359 & 0.31 & 226066 & 0.50 \\
\hline- & Taraxasterol & 6422306 & 3.09 & 3910398 & 8.61 \\
\hline- & n.i. & 565285 & 0.27 & 128466 & \\
\hline
\end{tabular}

Oxygenated monoterpenes

Sesquiterpenes hydrocarbons

11.6

14.0

Oxygenated sesquiterpenes

14.3

13.7 
Oxygenated diterpenes

Oxygenated triterpenes

Alkanes

Other compounds

Total identified compounds
1.1

7.3

43.5

16.1

96.3
1.2

17.9

26.3

18.6

94.8

${ }^{a}$ n.i. $=$ non identified; ${ }^{b}$ n.d. $=$ non detected 
Table 2. Quantitative Composition (mg/g) of Supercritical Extract of Calendula Officinalis

\begin{tabular}{|c|c|c|}
\hline Compound & $\mathrm{CO}_{2}$ & Ethanol- $\mathrm{CO}_{2}$ \\
\hline Thymol & 0.60 & 0.16 \\
\hline Carvacrol & 0.24 & 0.07 \\
\hline$\alpha$-Cubebene & 0.18 & 0.05 \\
\hline$\alpha$-Copaene & 0.42 & 0.12 \\
\hline$\beta$-Cubebene & 0.27 & 0.05 \\
\hline$\beta$-Gurjunene & 0.21 & 0.03 \\
\hline$\beta$-Humulene & 0.04 & 0.04 \\
\hline Alloaromadendrene & 0.39 & 0.07 \\
\hline y-Muurolene & 0.39 & 0.11 \\
\hline$\beta$-Selinene & 0.18 & 0.02 \\
\hline$(+)$-Ledene & 1.19 & 0.25 \\
\hline$\alpha$-Muurolene & 0.88 & 0.23 \\
\hline$\gamma$-Cadinene & 2.89 & 1.00 \\
\hline$\delta$-Cadinene & 3.02 & 0.86 \\
\hline Cadina-1(2),4-diene & 0.67 & 0.06 \\
\hline$\alpha$-Cadinene & 0.54 & 0.13 \\
\hline$\alpha$-Calacorene & 0.20 & 0.03 \\
\hline Viridifloror & 1.11 & 0.14 \\
\hline 1-10-di-epi-cubenol & 0.29 & 0.08 \\
\hline$\delta$-Cadinol & 0.27 & 0.05 \\
\hline Cubenol & 0.29 & 0.07 \\
\hline$\tau$-Cadinol & 3.56 & 0.84 \\
\hline n.i. ${ }^{a}$ Oxygenated sesquiterpene & 0.38 & 0.09 \\
\hline$\beta$-Eudesmol & 0.75 & 0.15 \\
\hline$\alpha$-Cadinol & 4.30 & 0.87 \\
\hline Nonadecane & 1.02 & 0.21 \\
\hline Eicosane & 0.25 & 0.04 \\
\hline Heneicosane & 1.81 & 0.30 \\
\hline Docosane & 0.18 & 0.03 \\
\hline Tricosane & 2.62 & 0.41 \\
\hline Tetracosane & 0.34 & 0.05 \\
\hline
\end{tabular}




\begin{tabular}{|c|c|c|}
\hline Pentacosane & 4.64 & 0.64 \\
\hline Hexacosane & 0.38 & 0.03 \\
\hline Heptacosane & 8.55 & 0.91 \\
\hline Octacosane & 1.19 & 0.14 \\
\hline Nonacosane & 14.27 & 1.67 \\
\hline Triacontane & 2.13 & n.d. ${ }^{b}$ \\
\hline Hentriacontane & 10.83 & 1.69 \\
\hline$\alpha$-Tocopherol & 1.29 & 0.69 \\
\hline Dotriacontane & 0.18 & n.d. \\
\hline n.i. sterol & 2.65 & n.d. \\
\hline n.i. sterol & 11.00 & 8.16 \\
\hline n.i. sterol & 30.48 & 10.74 \\
\hline$\beta$-Amyrenone & 1.20 & 0.54 \\
\hline$\beta$-Amyrin & 6.08 & 2.97 \\
\hline n.i. sterol & 8.99 & 4.35 \\
\hline$\alpha$-Amyrin + Lupeol & 8.96 & 4.34 \\
\hline n.i. oxygenated triterpene & 1.33 & 0.47 \\
\hline Taraxasterol & 13.29 & 8.09 \\
\hline Oxygenated monoterpenes & 0.8 & 0.2 \\
\hline Sesquiterpenes hydrocarbons & 11.5 & 3.1 \\
\hline Oxygenated sesquiterpenes & 10.9 & 2.3 \\
\hline Oxygenated triterpenes & 85.5 & 40.4 \\
\hline Alkanes & 48.6 & 6.1 \\
\hline Total quantitated compounds & 156.9 & 52.1 \\
\hline
\end{tabular}

${ }^{a}$ n.i. $=$ non identified; ${ }^{b}$ n.d. $=$ non detected 
Table 3. Bioaccessibility (\%) of Supercritical Extract of Calendula Officinalis

\begin{tabular}{lcc}
\hline Compound & Chemical group $^{a}$ & Bioaccessibility \\
\hline -Cadinene & SH & $79.3 \pm 9.7$ \\
$\delta$-Cadinene & SH & $87.9 \pm 11.8$ \\
$\tau$-Cadinol & OS & $75.1 \pm 3.4$ \\
a-Cadinol & OS & $87.1 \pm 3.7$ \\
Nonadecane & HC & $48.1 \pm 2.0$ \\
Tricosane & HC & $57.2 \pm 11.0$ \\
Pentacosane & HC & $48.3 \pm 2.8$ \\
Heptacosane & HC & $13.8 \pm 2.4$ \\
$\alpha$-Tocopherol & OT & $71.1 \pm 6.5$ \\
$\beta$-Amyrin & OT & $79.8 \pm 4.8$ \\
$\alpha$-Amyrin + Lupeol & OT & $82.1 \pm 8.8$ \\
Taraxasterol & OT & $75.5 \pm 2.1$ \\
& & $83.6 \pm 10.8$ \\
Sesquiterpenes hydrocarbons & & $81.1 \pm 3.6$ \\
Oxygenated sesquiterpenes & & $77.1 \pm 2.9$ \\
Oxygenated triterpenes & & $41.8 \pm 3.5$ \\
Alkanes & & \\
\hline
\end{tabular}

${ }^{a} \mathrm{SH}=$ sesquiterpene hydrocarbon, $\mathrm{OS}=$ oxygenated sesquiterpene, $\mathrm{HC}=$ alkanes, $\mathrm{OT}=$ oxygenated triterpene 
Figure 1.

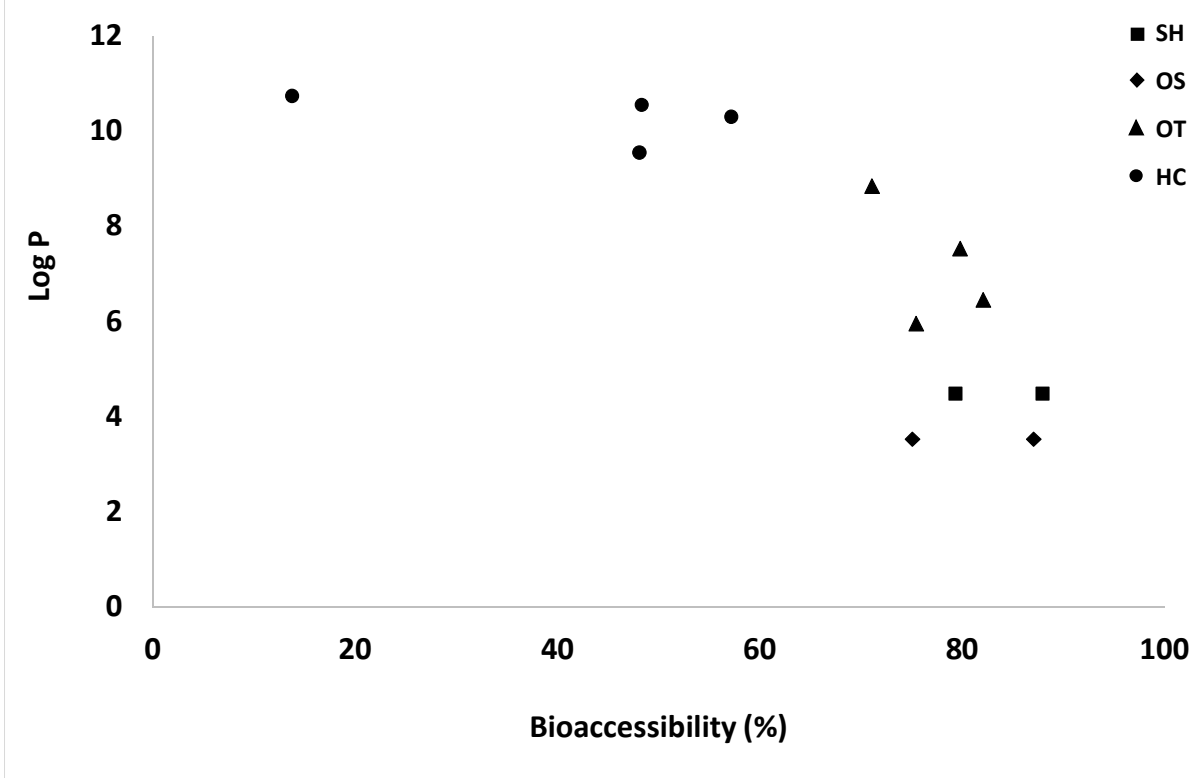


Figure 2.

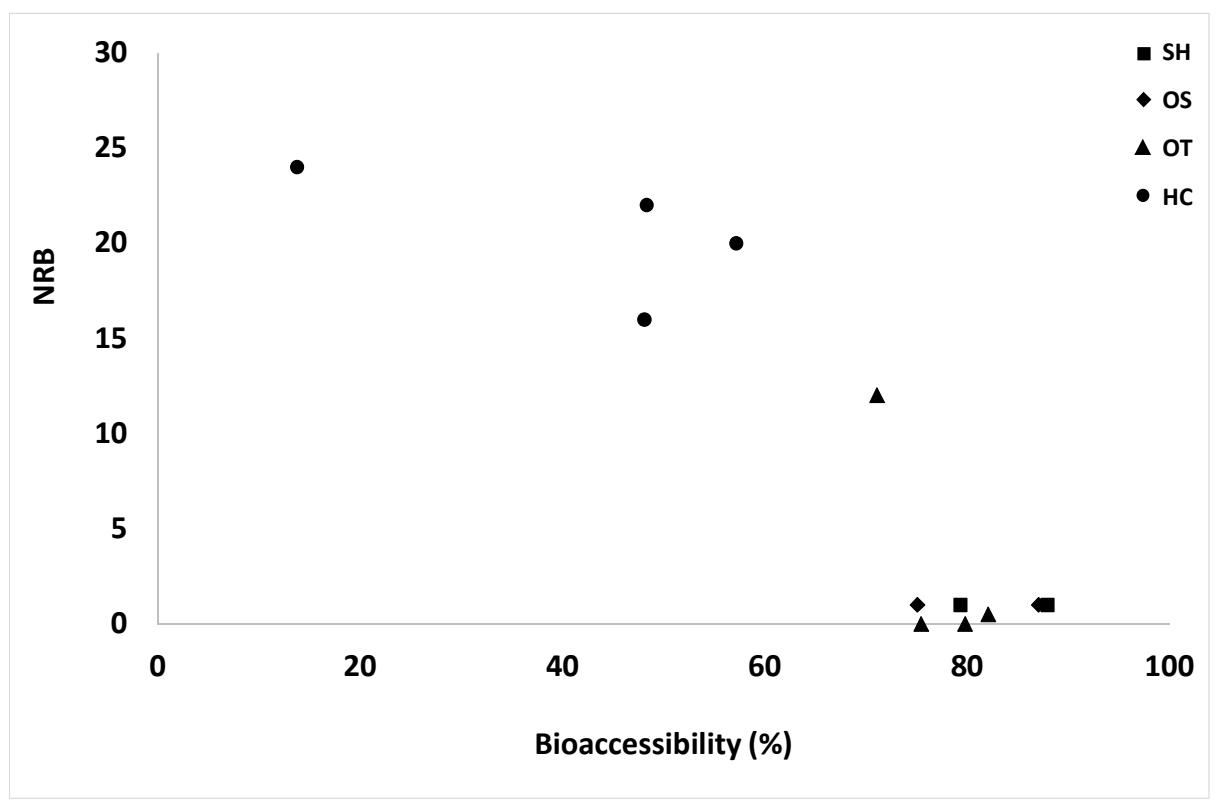


Figure 3.

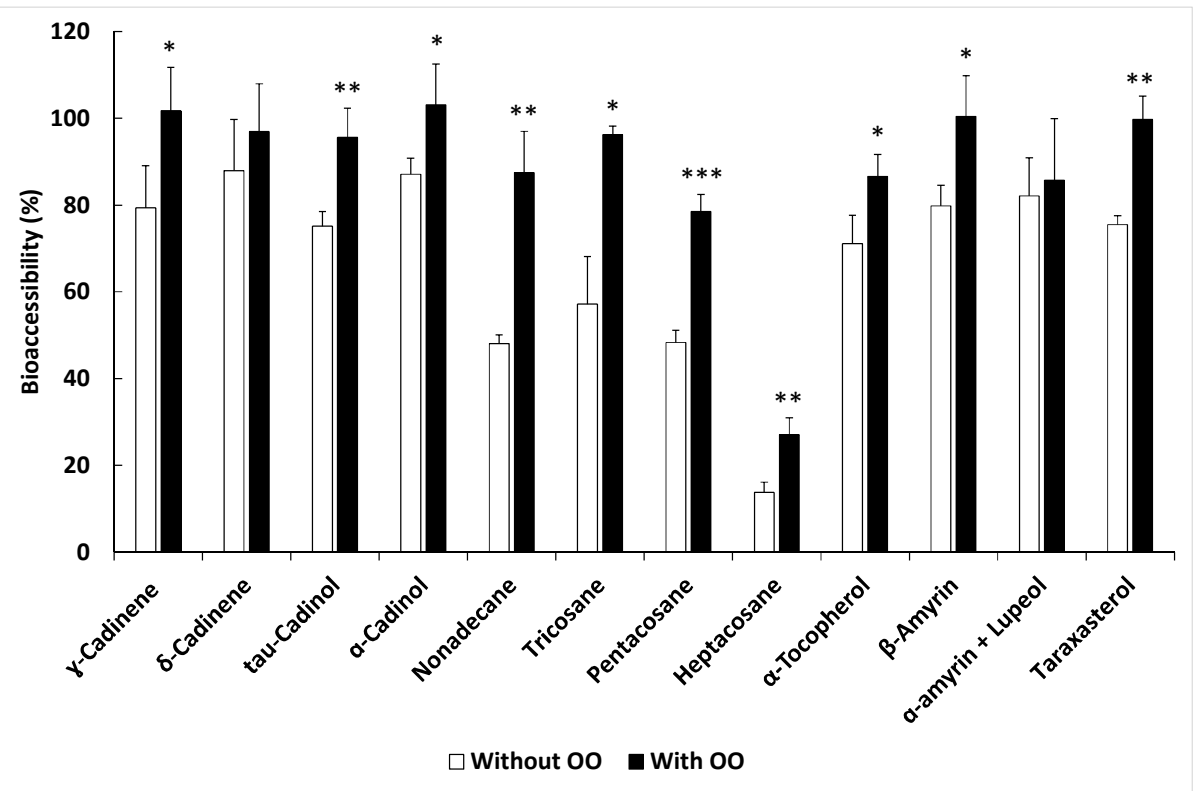

Bars within the same compound are significantly different if $\mathrm{p} \leq 0.05\left({ }^{*}\right), \mathrm{p} \leq 0.01\left({ }^{* *}\right)$ or $\mathrm{p} \leq 0.001(* * *)$. 
Figure 4.

a)

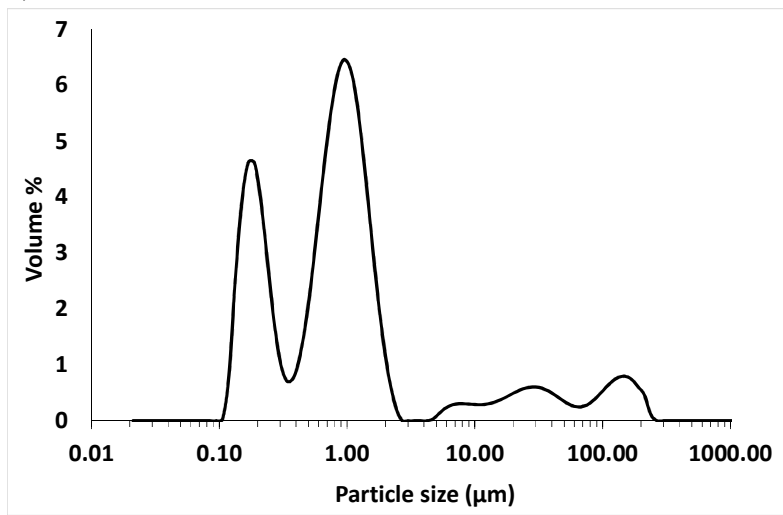

c)

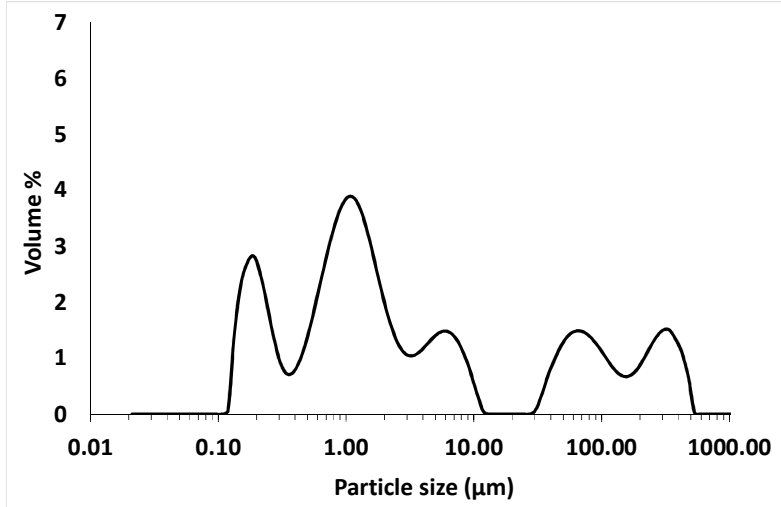

b)

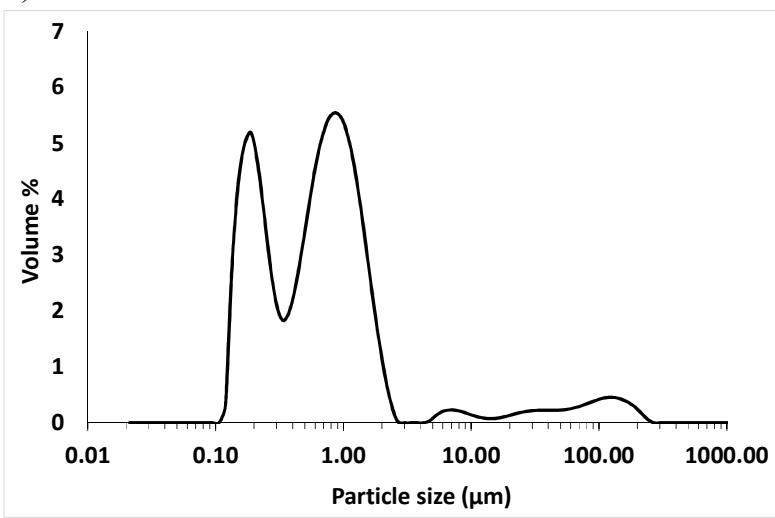

d)

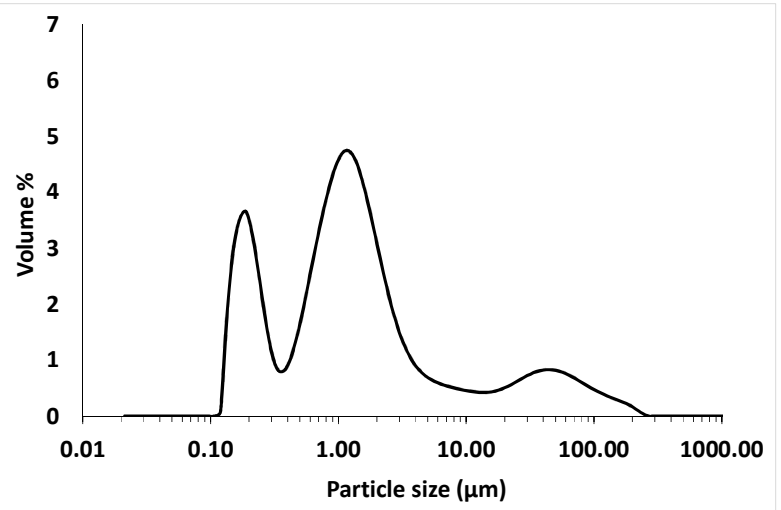

Isolated aqueous micellar phase after in vitro digestion of a) without marigold and without olive oil, b) without marigold and with olive oil, c) with marigold and without olive oil, and d) with marigold and with olive oil 
Figure 5.

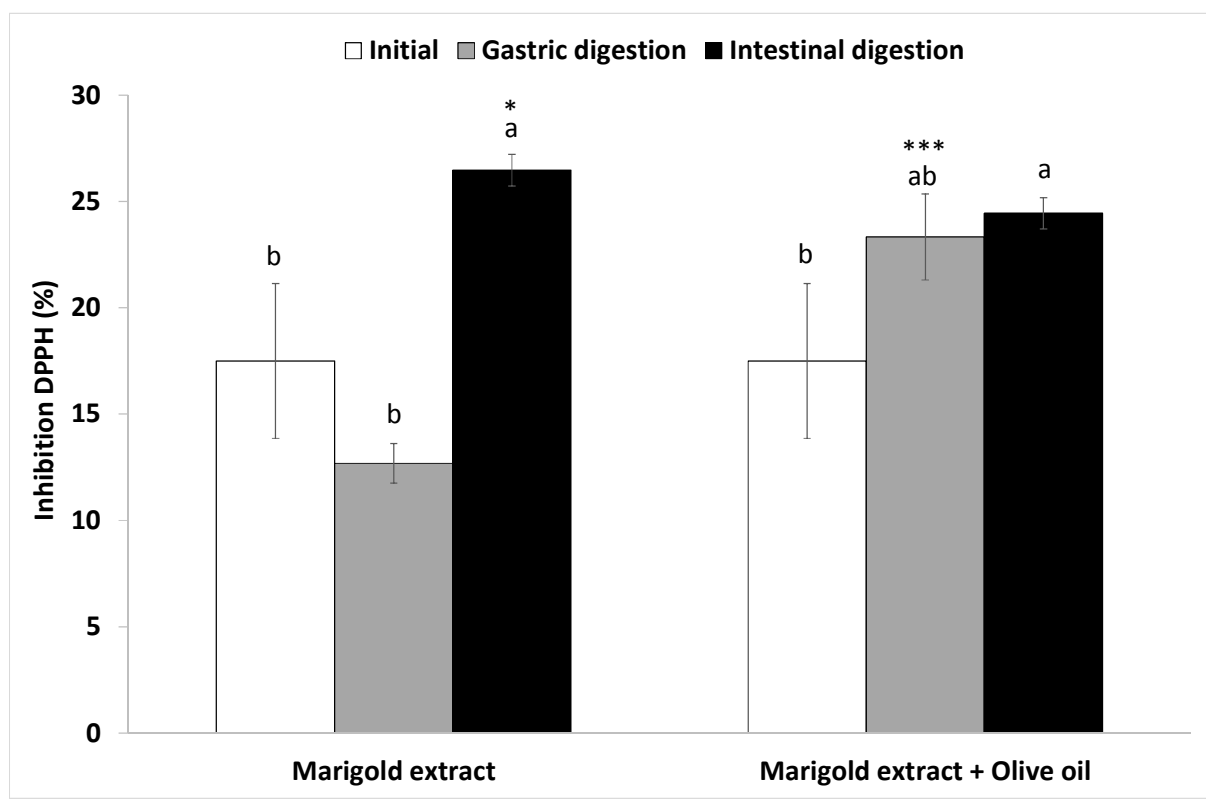

Different letters within the same treatment are significantly different. Bars within the same color are significantly higher if $\mathrm{p} \leq 0.05(*)$ or $\mathrm{p} \leq 0.001(* * *)$. 


\section{GRAPHIC FOR TABLE OF CONTENTS}

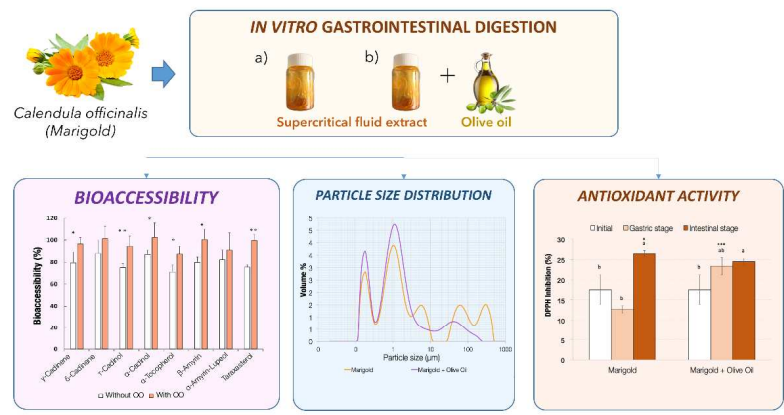

